\title{
Context effects in embodied lexical-semantic processing
}

\author{
Wessel O. van Dam*, Shirley-Ann Rueschemeyer, Oliver Lindemann and Harold Bekkering
}

Donders Institute for Brain, Cognition and Behavior, Radboud University Nijmegen, Netherlands

Edited by:

Anna M Borghi, University of Bologna Italy

\section{Reviewed by:}

Veronique Boulenger, Centre National de la Recherche Scientifique, France Rutvik Desai, Medical College of Wisconsin, USA

\section{*Correspondence:}

Wessel O. van Dam, P.O. Box 9104 6500 HE Nijmegen, Netherlands. e-mail:w.vandam@donders.ru.nl
The embodied view of language comprehension proposes that the meaning of words is grounded in perception and action rather than represented in abstract amodal symbols. Support for embodied theories of language processing comes from behavioral studies showing that understanding a sentence about an action can modulate congruent and incongruent physical responses, suggesting motor involvement during comprehension of sentences referring to bodily movement. Additionally, several neuroimaging studies have provided evidence that comprehending single words denoting manipulable objects elicits specific responses in the neural motor system. An interesting question that remains is whether action semantic knowledge is directly activated as motor simulations in the brain, or rather modulated by the semantic context in which action words are encountered. In the current paper we investigated the nature of conceptual representations using a go/no-go lexical decision task. Specifically, target words were either presented in a semantic context that emphasized dominant action features (features related to the functional use of an object) or non-dominant action features. The response latencies in a lexical decision task reveal that participants were faster to respond to words denoting objects for which the functional use was congruent with the prepared movement. This facilitation effect, however, was only apparent when the semantic context emphasized corresponding motor properties. These findings suggest that conceptual processing is a contextdependent process that incorporates motor-related knowledge in a flexible manner.

Keywords: embodiment, semantics, action, conceptual flexibility

\section{INTRODUCTION}

According to an embodied view of language comprehension, language concepts are grounded in motor and perceptual systems (Glenberg, 1997; Barsalou, 1999; Pulvermueller, 1999; Barsalou et al., 2003). Lexical-semantic representations are postulated to rely on sensori-motor brain areas and to reflect real-world experience with words' referents. For example an object such as a hammer is experienced by most people visually (i.e., we know what hammers look like) and motorically (i.e., we know what it feels like to wield a hammer). Thus, embodied language theories postulate that upon encountering the word hammer, experiential traces stored in modality specific (e.g., visual and motor) brain areas are activated. Importantly, these activations are seen to contribute to the lexical-semantic meaning of the word form hammer. This view differs fundamentally from symbolic (disembodied) accounts, in which conceptual representations are symbolic and amodal, and lexical-semantic meaning independent of real-world experience (Kintsch, 2008).

Converging evidence for the idea that sensory-motor brain areas are involved in language comprehension comes from behavioral, electrophysiological, and neuroimaging studies. Glenberg and Kaschak (2002) present the action-sentence compatibility effect (ACE), in which reading a sentence that implies an action toward or away from the body (e.g., open the drawer/close the drawer) facilitates a congruent action (i.e., moving a hand toward or away from the body). The authors argue that responses are facilitated because comprehending language about action recruits the same neural resources as required for action execution; thus comprehending the sentence primes a congruent motor act. Zwaan and
Taylor (2006) have shown that motor resonance is activated on-line during sentence comprehension. For example, while reading the sentence The marathon runner opened the water bottle, evidence for motor resonance is seen in conjunction with presentation of the verb opened. Furthermore motor resonance is elicited by words which unambiguously specify kinematic properties of actions, even if these words are not verbs (Taylor et al., 2008). For example, in the text He looked at the pie and turned the oven dial. The baking time needed to be shorter/longer evidence for motor resonance is seen in conjunction with the sentence final adjective (which specifies how the oven dial should be turned) rather than in conjunction with the verb turned.

In addition to words and sentences describing active events, isolated words denoting manipulable objects have also been shown to interact with motor behaviors and to activate neural motor areas. For example, Glover et al. (2004) show that words denoting objects that afford particular actions are sufficient to activate motor representations. Participants in their study were primed with the names of objects of different sizes (e.g., apple, grape) and required to reach out and grasp a wooden block. Interestingly, the maximum grip aperture during the reach (i.e., maximum distance between forefinger and thumb) was influenced by the size of the object denoted by the prime word, despite the fact that this information was irrelevant for executing the grasping task. Similarly, Rueschemeyer et al. (2010a) had participants perform a lexical decision task to words denoting objects typically brought toward or away from the body for functional use (e.g., cup or key, respectively). The authors found that responses to words were facilitated if the required response action was congruent to the 
action typically performed on the word's referent (i.e., responses to cup were faster if a movement was made toward the body rather than away from the body). This indicates that very specific information about how an object is manipulated is activated during lexical retrieval.

Evidence in favor of embodied theories of language also comes from recent neuroimaging studies. Specifically, the comprehension of action verbs (Kemmerer et al., 2008), action sentences (Desai et al., 2010), and words denoting manipulable objects (Chao and Martin, 2000; Saccuman et al., 2006; Rueschemeyer et al., 2010b) all reliably activate the cerebral motor system. Furthermore, embodied lexical-semantic representations activate the neural motor system in a somatotopic manner (Hauk et al., 2004; Tettamanti et al., 2005; Aziz-Zadeh et al., 2006; but see also de Zubicaray et al., 2010). Findings from the studies reviewed above provide strong evidence for functional links between the neural motor system and lexical-semantic processing of words that entail a motor component.

It has been suggested that embodied lexical-semantic representations are fast, automatic and invariant. Pulvermueller et al. (2000) demonstrated that category-specific activation can be observed as early as about $200 \mathrm{~ms}$ after word onset (see also Hauk and Pulvermueller, 2004), and occurs irrespective of attention to action words (Shtyrov et al., 2004; Pulvermueller et al., 2005). Pulvermueller proposes that strong functional links between language and motor systems have developed as a consequence of the fact that actions and their referents often co-occur near-simultaneously. Specifically, the action and the word co-occur frequently, and thereby, neural populations recruited for processing a word form and those involved in processing the referent body movement frequently fire together and become strongly linked (Pulvermueller, $1999,2001)$. Due to the strong within-assembly connections that link language and action representations, action word recognition will thereby automatically and invariably trigger activation in specific action-related networks.

However, in contrast to what would be expected if embodied representations are indeed automatic and invariant, several studies have failed to find motor-related activity for words with an action-semantic component (Rueschemeyer et al., 2007; Raposo et al., 2009). Raposo et al. (2009) showed in a recent fMRI study that action verbs in isolation (e.g., kick) or in literal sentences (e.g., kick the ball) elicit a response in motor/premotor cortices. Action verbs in an idiomatic context (e.g., kick the bucket), however, did not elicit such activations. These findings strongly challenge the automaticity of motor-related activity for action words and rather suggests that the activation of meaning attributes of words is a flexible and contextually dependent process (but see also Boulenger et al., 2009). In a similar vein, Rueschemeyer et al. (2007) showed that processing of morphologically complex verbs built on motor stems showed no differences in involvement of the motor system when compared with processing complex verbs with abstract stems. For example, the difference between the verb begreifen (to comprehend) vs. bedenken (to think). In the first case the morphologically complex verb is a prefixed form of the simple motor verb greifen (to grasp), whereas in the latter case it is a prefixed form of the abstract verb denken (to think). All these studies provide evidence that motor responses rather than being automatic and invariable, depend on the context (i.e., sentence context in the case of Raposo et al., 2009, and morphological context in the case of Rueschemeyer et al., 2007) in which action words are encountered. That is, a crucial factor for observing activity in motor and premotor regions during action word processing seems to be that the context emphasizes motor properties (suggesting that representations are flexible).

The failure of some studies to report motor activation for actionrelated words embedded in various contexts already speaks against a strictly automatic interpretation, and suggests that motor activations may be called on in a flexible manner during word processing. In the two studies cited above, motor information is not useful in comprehending the given language utterances (i.e., idiomatic phrases or morphologically complex words), and indeed the results indicate that in these cases the motor system is not reliably activated. However, even in cases in which motor information is helpful in processing semantic content, a certain degree of flexibility on the level of specific motor programs might be expected. For example, the motor programs associated with the word cup are quite different in the sentences She filled her cup at the tap/She drank from the cup. In the first case, the actor in the sentence brings the cup away from her body and toward the tap, in the second she brings the cup toward her mouth. Previous research has shown that words referring to manipulable objects activate motor areas (Chao and Martin, 2000; Saccuman et al., 2006; Rueschemeyer et al., 2010a), but since most objects can be used in multiple ways, does the motor contribution to lexical-semantic comprehension also vary depending on the language context in which a word is presented?

In the current study, we investigated precisely this issue: within a given modality (i.e., action) we investigated whether a word always activates a specific motor program, or whether the motor program activated depends on the context in which a word is presented. Previously we (Rueschemeyer et al., 2010a) showed that comprehension of words denoting manipulable objects (e.g., cup, ham$m e r$ ) are facilitated by the prior planning of an action congruent to the prototypical use of the object. In other words, participants were faster to respond to the word cup when they had planned an action toward their body (the most common use of the cup being a vessel to drink from) than when they had planned an action away from their body; the opposite pattern was true for words denoting objects typically brought away from the body (e.g., hammer). We thus show a strong congruency effect between the typical action associated with a word's referent and processing of the individual word. The target words in this previous study were presented in isolation, i.e., without any explicit language context. In the current study we investigated whether embedding the same words in a lexical context suggesting a non-prototypical (but not unfamiliar) use of the objects would influence the observed congruency effects. To this end object words were always preceded by a prime word providing a context that emphasized either the dominant action feature (thirst-cup) or a non-dominant action feature $(\operatorname{sink}-\boldsymbol{c u p})$. As previous studies have shown that the retrieval of a particular conceptual feature depends on the context in which a word is encountered, we hypothesized that the effect of movement preparation on word processing would interact with the 
semantic context in which a word is encountered. Specifically, in trials in which the semantic context emphasizes the dominant motor properties of a concept, we expect faster onset latencies to words denoting objects for which the functional use is congruent with the prepared movement in directionality. Furthermore, we expect that this facilitation effect is not present if the semantic context emphasizes motor properties of a concept that are not related to the object's functional use.

\section{EXPERIMENT \\ METHOD \\ Participants}

Thirty-five subjects participated; the average age was 21.3 years. All subjects were students at the Radboud University Nijmegen and participated either for money or course credit. No subject was aware of the purpose of the experiment.

\section{Stimuli}

Words were presented in white Arial fonts on a black background. The average word length was 10 letters. For a viewing distance of $100 \mathrm{~cm}$, the stimuli subtended on average a visual angle of $0.57^{\circ} \times 2.86^{\circ}$. A total of 120 letter string stimuli were created for the experiment (stimuli with English translations can be seen in Table 1). Eighty were real Dutch words denoting familiar objects and comprised the critical experimental stimuli. The remaining 40 stimuli were Dutch pseudowords (i.e., phonotactically and orthographically legal letter strings with no meaning in Dutch). Target stimuli belonged to one of two experimental conditions: (1) words denoting objects for which the functional use is associated with a movement toward the body (e.g., telephone, photo camera), and (2) words denoting objects for which the functional use is associated with a movement away from the body (e.g., hammer, pencil). Target stimuli were presented in two contexts: (1) target words were preceded by a word that emphasized the action feature related to the functional use (e.g., conversation-telephone, nail-hammer), and (2) target words were preceded by a word that emphasized an action feature not related to the functional use (e.g., adapter-telephone, tool belt-hammer).

To test that stimuli were truly matched with regards to important psycholinguistic variables, a questionnaire was administered to 15 native Dutch speakers who did not participate in the behavioral experiment (see Table 2 for results). In this questionnaire, participants were asked to rate words on a 7-point scale with respect to (1) the imageability of the noun ( $1=$ very difficult to imagine the referent noun, 7 = very easy to imagine the referent noun), (2) whether the noun denoted an object that you typically bring toward or away from the body $(-3=$ toward the body, $+3=$ away from the body).

The results of the questionnaire showed that nouns were matched across the two conditions with respect to imageability (body: $M=6.82, \mathrm{SE}=0.024$; world: $M=6.76, \mathrm{SE}=0.029), t(1,78)=1.46$, $p>0.1$. For object nouns, participants agreed that body words referred to objects that you typically bring toward the body, world words referred to objects you typically bring away from the body (body: $M=-1.13, \mathrm{SE}=0.049$; world: $M=2.33$, $\mathrm{SE}=0.057$ ), both means significantly differed from 0 as indicated by one-sample
Table 1 | Dutch words associated with a movement toward the body (body words) and a movement away from the body (world words).

English translations are printed in italics.

\begin{tabular}{|c|c|c|c|}
\hline Body words & & World words & \\
\hline Haarband & Hair ribbon/Hairband & Zwabber & Swab/Mob \\
\hline $\mathrm{La}$ & Drawer & Spaarpot & Money box \\
\hline Sjaal & Scarf & Spade & Spade/Shovel \\
\hline Microfoon & Microphone & Zaag & Saw \\
\hline Loep & Magnifying glass & Vaas & Vase \\
\hline Hoed & Hat & Naald & Needle \\
\hline Nagelvijl & Nail file & Kaars & Candle \\
\hline Pleister & Band-Aid & Plant & Plant \\
\hline Fluit & Flute & Flesopener & Bottle opener \\
\hline Bril & (pair of) Spectacles & Deegroller & Rolling pin \\
\hline Wijnglas & Wine glass & Koekenpan & Frying pin \\
\hline Mok & Mug & Voetbal & Football \\
\hline Make-up & Make up & Theepot & Teapot \\
\hline Zakdoek & Handkerchief & Speld & Pin \\
\hline Lolly & Lollipop & Stempel & Stamp \\
\hline Halssnoer & Necklace & Sleutel & Key \\
\hline Helm & Helmet & Lamp & Lamp \\
\hline Telefoon & Telephone & Schep & Scoop/Shovel \\
\hline Shampoo & Shampoo & Knikker & Marble \\
\hline Armband & Bracelet & Bijl & Axe \\
\hline Tondeuse & (pair of) Clippers & Baksteen & Brick \\
\hline Mondharmonica & Mouth organ & Fakkel & Torch \\
\hline Want & Toothbrush & Bel & Bell \\
\hline Tandenborstel & Mitten & Hamer & Hammer \\
\hline Handdoek & Towel & Computer & Computer \\
\hline Ring & Ring & Hengel & Fishing rod \\
\hline Trompet & Trumpet & Pen & Pen \\
\hline Schoen & Shoe & Boor & Drill \\
\hline Lippenstift & Lipstick & Gloeilamp & Lightbulb \\
\hline Lepel & Spoon & Trommel & Drum \\
\hline Oorbel & Earring & Kapstok & Coat rack \\
\hline Borstel & Brush & Verfpot & Pot/Tin of paint \\
\hline Verrekijker & Binoculars & Vergiet & Stainer/Colander \\
\hline Stropdas & Tie/Necktie & Karaf & Decanter/Carafe \\
\hline Gordel & Belt & Garde & Whisk \\
\hline Vork & Fork & Paraplu & Umbrella \\
\hline Parfum & Perfume & Mes & Knife \\
\hline Horloge & Watch & Potlood & Pencil \\
\hline Jas & Jacket & Ventilator & Fan \\
\hline Fototoestel & Photo camera & Dobbelsteen & Dice \\
\hline
\end{tabular}

$t$-tests (all $p$-values $<0.001$ ). In order to obtain an objective measure of word frequency, we calculated the mean lemma frequency per million for each condition using the lexical database (Baayen et al., 1993). This gave a mean of $567(\mathrm{SE}=128.3)$ for the body words and $487(\mathrm{SE}=119.3)$ for the world words. An independent sample $t$-test indicated words were matched on frequency, $t(1,78)=1.48$, $p>0.1$. Additionally, independent sample $t$-tests indicated that nouns were matched with regard to length (body: $M=6.8$, world: $M=6.3), t(1,78)=0.90, p>0.2$. 


\section{Procedure and design}

Participants were seated comfortably in front of a computer monitor, and responded by means of a key press (i.e., by pressing either a key that was located nearer or further from the body). To start a trial participants had to press the start button of a response device (located in the middle of the response device). Subsequently, they received a cue (i.e., the letters A or B) that signaled them to prepare a movement (either toward or away from the body) which they only executed if the second word was lexically valid. Participants were instructed to read both words carefully. The advent of the two words was signaled by a fixation cross (appearing centrally for $500 \mathrm{~ms}$ ). The first word was presented for $1000 \mathrm{~ms}$. The second word appeared $1000 \mathrm{~ms}$ after the offset of the first word, calling for a speeded response to the identity of the word (i.e., a response in the case that the second word was a real word in Dutch). The second word remained visible until participants responded, or for a maximum of $2000 \mathrm{~ms}$ (see Figure 1 for an illustration of a Go and NoGo trial). That is, the first presented word was not clearly associated with a specific direction or action, the differences we report are relative differences between reaction times (RTs) acquired in response to the second presented word. The experiment comprised 160 critical trials composed by 20 replications of the factorial combination of two movement cues, two word types, and two contexts. That is, the direction of the participant's response and the motor program generally associated with the word's referent either corresponded or not (action congruent vs. action incongruent) and the word was either presented in a language context highlighting the functional use or a less typical use of the object (focus on dominant vs. non-dominant action feature). The order in which word pairs were presented was counterbalanced over participants. That is, half of the participants first saw a target word (e.g., telephone) preceded by a word that emphasized the dominant action feature (conversation) and then the same target word preceded by a word that emphasized a nondominant action feature (adapter) and vice versa.

\section{Data analysis}

We measured the latencies to recognize that a presented Dutch word was lexically valid defined as the time difference between word onset and release of the start button. Average RTs can be seen in Figure 2. Additionally, we recorded movement times (MTs; i.e., the time from releasing the start button until depressing the target button). Trials with extreme RTs or MTs $(2 \times$ STD \pm mean $)$ were treated as outliers and excluded from further analysis. This led to an exclusion of $9.12 \%$ of the data. The significance criterion for all analyses was set to $\alpha=0.05$.

\section{RESULTS}

The error rates in the lexical decision task were on average lower than $2 \%$ and therefore not further analyzed. RTs were averaged for each participant in each condition (see Table 3 for means) and submitted to a two-way repeated measures analysis of variance (ANOVA) with factors Action Congruency (congruent vs. incongruent) and Context (focus on dominant vs. non-dominant action feature). The main effect for Action Congruency and Context did not reach significance, both $p$-values $>0.2$. Importantly, in line with our hypothesis, the ANOVA revealed a significant interaction $F(1,34)=5.76, p<0.05, \eta_{p}^{2}=0.145$, indicating that the RT differences between action congruent and action incongruent words were modulated by the different context conditions. To further explore this interaction, we calculated post hoc paired sample $t$-tests. When the context emphasized the dominant action feature, words congruent with the prepared action were processed faster than incongruent words, $t(34)=-2.21, p<0.05$. However, if the context focused on non-dominated action features, the mean RTs to action congruent and action incongruent words did not differ statistically, $t(34)<1$, and the RT pattern reversed descriptively.

Movement times were averaged for each participant in each condition and submitted to a two-way repeated measures ANOVA. The main effect for Action Congruency and Context did not reach significance, both $p$-values $>0.05$. Furthermore, the ANOVA did not reveal a significant interaction $(p>0.5)$.

\section{Go trial NoGo trial}

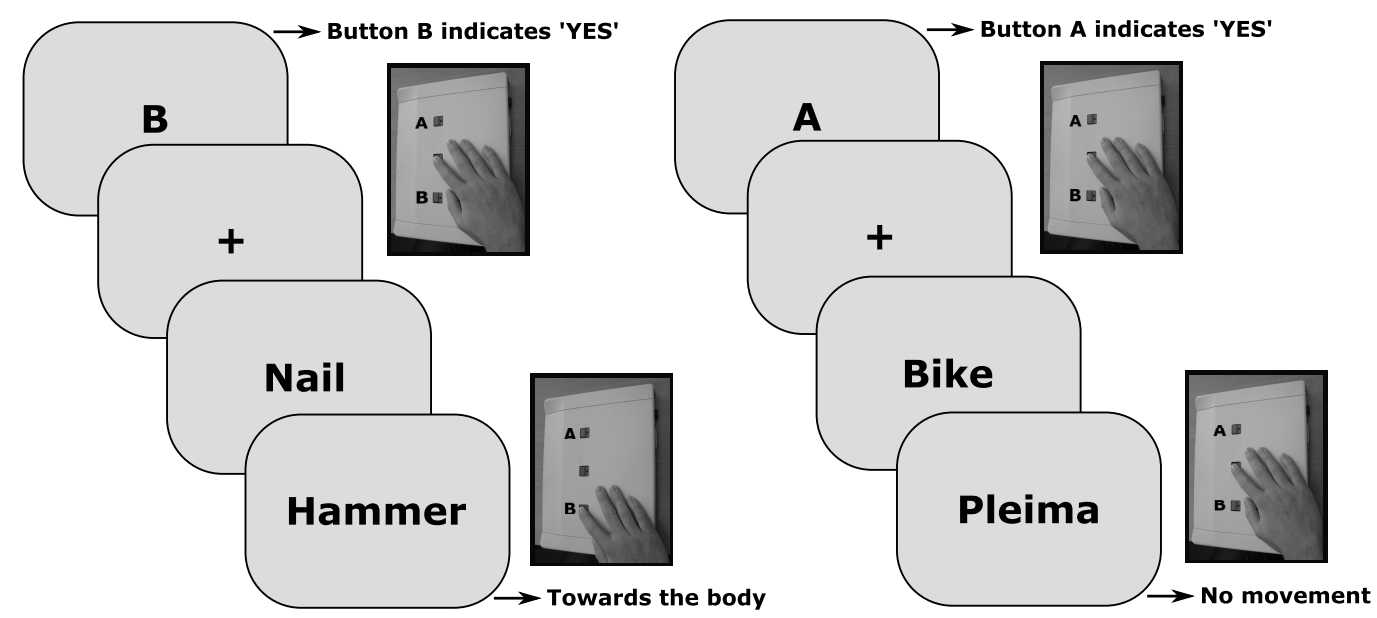

FIGURE 1 | Illustration of a Go and NoGo trial. 


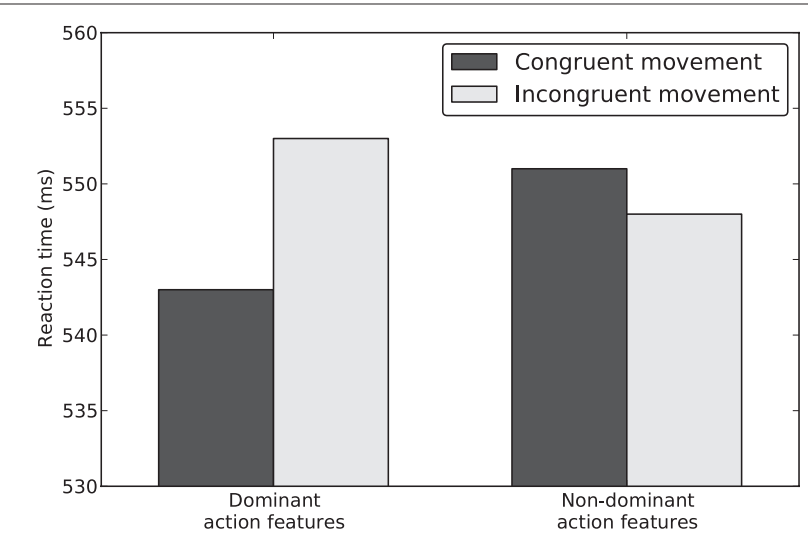

FIGURE 2 |Average reaction times (RTs) for words, as a function of the congruency between the cue and associated movement direction, and the contextual focus (focus on dominant action feature vs. non-dominant action feature).

Table 2 | Mean ratings of the pre-tests.

\begin{tabular}{lll}
\hline & Body words & World words \\
\hline Length & 6.8 & 6.3 \\
Lemma frequency & 567 & 487 \\
per million (CELEX) & & 6.76 \\
Imageability & 6.82 & 2.33 \\
Action association & -1.33 & \\
\hline
\end{tabular}

Table 3 |Average performance rates (PR), reaction times (RTs), and movement times (MTs) with standard errors for congruent and incongruent trials in both the dominant focus and non-dominant focus condition.

PR (SE) $\quad$ RT (SE) $\quad$ MT (SE)

\begin{tabular}{|c|c|c|c|}
\hline \multicolumn{4}{|c|}{ DOMINANT FOCUS } \\
\hline Congruent & $98.4(0.26)$ & $543(14.73)$ & $276(16.05)$ \\
\hline Incongruent & $98.3(0.37)$ & $553(16.21)$ & $272(15.55)$ \\
\hline \multicolumn{4}{|c|}{ NON-DOMINANT FOCUS } \\
\hline Congruent & $98.6(0.35)$ & $551(15.54)$ & $272(15.51)$ \\
\hline Incongruent & $98.3(0.37)$ & $548(15.84)$ & 269 (15.69) \\
\hline
\end{tabular}

\section{DISCUSSION}

The present study clearly demonstrates contextual effects on embodied word processing, evidenced by a reliable Action Congruency $\times$ Context interaction effect. Specifically, a congruency effect (i.e., faster word recognition times) is observed for trials in which the direction of the participant's response and the motor program generally associated with the word's referent correspond, but only if the word is presented in a language context highlighting the functional use of the object (e.g., thirst - cup). In a language context highlighting a less typical use of the object (e.g., sink - cup), the congruency effect disappears. These findings suggest that activation of modality specific (in this case motor) information during word comprehension is a flexible and contextually dependent process, and not a process of an automatic and invariant simulation of a specific motor program. In other words, semantic features are flexibly recruited with respect to the type of properties emphasized by the semantic context (see also Hoenig et al., 2008; Masson et al., 2008; Raposo et al., 2009).

The congruency effect in the present study extends the results of our previous research on motor involvement in language processing (Rueschemeyer et al., 2010a) and demonstrates the important role of contextual information in the embodied processing of language. We show that preparing an action congruent to the typical, functional use of an object facilitates processing of the word denoting the object. We suggest that words presented in isolation rely on a default representation, which highlights the typical functional use of the object. In our current results we extend this finding to show (not surprisingly) that words presented in a context highlighting the denoted object's functional use are also facilitated by preparing a congruent action.

Interestingly, the action congruency did not affect the word processing time if the semantic context emphasized action features not belonging to the core of the concept. There are two alternative explanations for this finding: first, it is possible that motor semantic features are not activated at all in a non-dominant action context. Alternatively, it is possible that dominant conceptual features are co-activated along with non-dominant features. In other words, cup in the context of sink activates both motor programs associated with moving a cup toward the sink and motor programs associated with bringing the cup toward the mouth. This activation of two opposite motor programs might have resulted in a null effect for trials in which properties unrelated to the functional use of the object are emphasized. In this scenario, the priming effect of movement preparation on lexical access is canceled out, because motor codes underlying two opposing movements are activated simultaneously. This explanation is in line with the findings of Hoenig et al. (2008) in which they show that activation in regions coding non-dominant object features increased if semantic context encouraged participants to focus on the non-dominant feature. However, brain regions coding information about dominant features were co-activated, even when not directly probed by the task. The authors argue that dominant object features are co-activated with non-dominant object features through collateral support (see also Farah and McClelland, 1991).

The reliable congruency effect for trials in which there was a contextual focus on action properties related to the functional use of the object, bolsters the claim that in processing of a word with an action-semantic component we activate information stored in modality specific sensory-motor systems (Hoenig et al., 2008). The present study, however, cannot determine whether motor system involvement is a fundamental necessity or a consequence of word comprehension. One might argue that the reason for observing a congruency effect between prepared movement direction and associated movement direction is because the person voluntarily images the functional use of the referent object, after the meaning of the object word is already understood. The design of our current study prevents us from ruling out this possibility. For a better understanding of the exact nature of the interaction between context 
and embodied word processing, we need to further investigate the temporal dynamics of the observed contextual effects on embodied word processing.

\section{CONCLUSION}

The current study demonstrates that the interaction between lexical-semantic processing and movement preparation processes was modulated by the context in which these words were encountered. Together the data suggest that context plays a fundamental

\section{REFERENCES}

Aziz-Zadeh,L., Wilson, S.M., Rizzolatti,G., and Iacoboni, M. (2006). Congruent embodied representations for visually presented actions and linguistic phrases describing actions. Curr. Biol. 16, 1818-1823.

Baayen, R., Piepenbrock, R., and Van Rijn, H. (1993). The CELEX Lexical Database (CD-ROM). Philadelphia, PA: Linguistic Data Consortium, University of Pennsylvania

Barsalou, L. W. (1999). Perceptual symbol systems. Behav. Brain Sci. 22, 577-660.

Barsalou, L. W., Simmons, W. K., Barbey, A. K., and Wilson, C. D. (2003). Grounding conceptual knowledge in modality-specific systems. Trends Cogn. Sci. 7, 84-91.

Boulenger,V.,Hauk, O., and Pulvermueller, F. (2009). Grasping ideas with your motor system: semantic somatotopy in idiom comprehension. Cereb. Cortex 19, 1905-1914.

Chao, L. L., and Martin, A. (2000). Representation of manipulable manmade objects in the dorsal stream. Neuroimage 12, 478-484.

Desai, R. H., Binder, J. R., Conant, L. L., and Seidenberg, M. S. (2010). Activation of sensory-motor areas in sentence comprehension. Cereb. Cortex 20, 468-478.

de Zubicaray, G., Postle, N., McMahon, K., Meredith, M., and Ashton, R. (2010). Mirror neurons, the representation of word meaning, and the foot of the third left frontal convolution. Brain Lang. 112, 77-84.

Farah, M. J., and McClelland, J. L. (1991). A computational model of semantic memory impairment: modality specificity and emergent category specificity. J. Exp. Psychol. Gen. 120, 339-357.
Glenberg, A. M. (1997). What memory is for. Behav. Brain Sci. 20, 1-55.

Glenberg, A. M., and Kaschak, M. (2002). Grounding language in action. Psychon. Bull. Rev. 9, 558-565.

Glover, S., Rosenbaum, D. A., Graham, J., and Dixon, P. (2004). Grasping the meaning of words. Exp. Brain Res. 154, 103-108.

Hauk, O., Johnsrude, I., and Pulvermueller, F. (2004). Somatotopic representation of action words in human motor and premotor cortex. Neuron 41, 301-307.

Hauk, O., and Pulvermueller, F. (2004). Neurophysiological distinction of action words in the fronto-central cortex. Hum. Brain Mapp. 21, 191-201.

Hoenig, K., Sim, E.-J., Bochev, V., Herrnberger, B., and Kiefer, M. (2008). Conceptual flexibility in the human brain: dynamic recruitment of semantic maps from visual, motor, and motion-related areas. J. Cogn. Neurosci. 20, 1799-1814.

Kemmerer, D., Castillo, J. G., Talavage, T., Patterson, S., and Wiley, C. (2008). Neuroanatomical distribution of five semantic components of verbs: evidence from fMRI. Brain Lang. 107, 16-43.

Kintsch, W. (2008). "Symbol systems and perceptual representations," in Symbols and Embodiment, eds M. De Vega, A. Glenberg, and A. Graesser (Oxford: Oxford University Press), 145-164.

Masson, M., Bub, D., and Warren, C. (2008). Kicking calculators: contribution of embodied representations to sentence comprehension. J. Mem. Lang. 59, 256-265.

Pulvermueller, F. (1999). Words in the brain's language. Behav. Brain Sci. 22, 253-336.

role in sensory-motor activations during language processing. That is, the activation of specific motor properties in language comprehension is flexible and context-dependent.

\section{ACKNOWLEDGMENTS}

The authors thank P. de Water, and G. van Oijen for technical support. The study was supported by NWO-Veni grant $\mathrm{nr}$ (016094-053) to Shirley-Ann Rueschemeyer and NWO-VICI grant nr (453-05-001) to Harold Bekkering.

Pulvermueller, F. (2001). Brain reflections of words and their meaning. Trends Cogn. Sci. 5, 517-524.

Pulvermueller, F., Härle, M., and Hummel, F. (2000). Neurophysiological distinction of verb categories. Neuroreport 11 2789-2793.

Pulvermueller, F., Shtyrov, Y., and Ilmoniemi, R. (2005). Brain signatures of meaning access in action word recognition. J. Cogn. Neurosci. 17, 884-892.

Raposo, A., Moss, H. E., Stamatakis, E. A., and Tyler, L. K. (2009). Modulation of motor and premotor cortices by actions, action words and action sentences. Neuropsychologia 47, 388-396.

Rueschemeyer, S.-A., Brass, M., and Friederici, A. D. (2007). Comprehending prehending: neural correlates of processing verbs with motor stems. J. Cogn. Neurosci. 19, 855-865.

Rueschemeyer, S.-A., Pfeiffer, C., and Bekkering, H. (2010a). Body schematics: on the role of the body schema in embodied lexical-semantic representations. Neuropsychologia 48, 774-781.

Rueschemeyer, S.-A., van Rooij, D., Lindemann, O., Willems, R., and Bekkering, H. (2010b). The function of words: distinct neural correlates for words denoting differently manipulable objects. J. Cogn. Neurosci. 22, 1844-1851.

Saccuman, M. C., Cappa, S. F., Bates, E.A. Arevalo, A., Della Rosa, P., Danna, M., and Perani, D. (2006). The impact of semantic reference on word class: an fMRI study of action and object naming. Neuroimage 32, 1865-1878.

Shtyrov, Y., Hauk, O., and Pulvermueller, F. (2004). Distributed neuronal networks for encoding category-specific semantic information: the mismatch negativity to action words. Eur. J. Neurosci. 19, 1083-1092.

Taylor, L. J., Lev-Ari, S., and Zwaan, R. A. (2008). Inferences about action engage action systems. Brain Lang. 107, 62-67.

Tettamanti, M., Buccino, G., Saccuman, M. C., Gallese, V., Danna, M., Scifo, P., Fazio, F., Rizzolatti, G., Cappa, S. F., and Perani, D. (2005). Listening to action-related sentences activates fronto-parietal motor circuits. J. Cogn. Neurosci. 17, 273-281.

Zwaan, R. A., and Taylor, L. J. (2006). Seeing, acting, understanding: motor resonance in language comprehension. J. Exp. Psychol. Gen. 135, 1-11.

Conflict of Interest Statement: The authors declare that the research was conducted in the absence of any commercial or financial relationships that could be construed as a potential conflict of interest.

Received: 16June 2010; paperpending published: 03 July 2010; accepted: 21 August 2010; published online: 04 October 2010. Citation: van Dam WO, Rueschemeyer $S-A$, Lindemann $O$ and Bekkering $H$ (2010) Context effects in embodied lexicalsemantic processing. Front. Psychology 1:150. doi: 10.3389/fpsyg.2010.00150

This article was submitted to Frontiers in Cognition, a specialty of Frontiers in Psychology.

Copyright (C) 2010 van Dam, Rueschemeyer, Lindemann and Bekkering. This is an openaccess article subject to an exclusive license agreement between the authors and the Frontiers Research Foundation, which permits unrestricted use, distribution, and reproduction in any medium, provided the original authors and source are credited. 